\title{
Optimizing the Treatment Options for Metastatic Castration Resistant Prostate Cancer in a Resource Poor Setting: A Single Centre Experience
}

\author{
Olufunmilade Omisanjo ${ }^{1,2, *}$, Olawale Ogunremi ${ }^{2}$, Olufemi Ojewuyi ${ }^{2}$, Fatai Balogun ${ }^{2}$, \\ Mofeyisayo Omorinde ${ }^{2}$, Stephen Ikuerowo ${ }^{1,2}$ \\ ${ }^{1}$ Department of Surgery, Lagos State University College of Medicine, Ikeja, Lagos, Nigeria \\ ${ }^{2}$ Department of Surgery, Lagos State University Teaching Hospital, Ikeja, Lagos, Nigeria
}

Email address:

sanjofunmi@yahoo.com (O. Omisanjo)

${ }^{*}$ Corresponding author

\section{To cite this article:}

Olufunmilade Omisanjo, Olawale Ogunremi, Olufemi Ojewuyi, Fatai Balogun, Mofeyisayo Omorinde, Stephen Ikuerowo. Optimizing the Treatment Options for Metastatic Castration Resistant Prostate Cancer in a Resource Poor Setting: A Single Centre Experience. International Journal of Clinical Urology. Vol. 3, No. 1, 2019, pp. 22-26. doi: 10.11648/j.ijcu.20190301.16

Received: May 18, 2019; Accepted: August 24, 2019; Published: September 16, 2019

\begin{abstract}
Background: Metastatic Castration Resistant Prostate Cancer (mCRPC) is invariably a terminal disease. Though international guidelines exist on $\mathrm{MCRPC}$ management, there are varied practices regarding the sequencing of limited available treatment options locally. Objectives: To describe the treatment sequence and outcome of management of mCRPC at the Lagos State University Teaching Hospital, Ikeja over a 4 year period. Methods: This was a retrospective study in which the clinical records of all patients diagnosed with mCRPC at the Lagos State University Teaching Hospital, Nigeria between June 2012 and June 2016 were retrieved and analyzed. Results: There were 30 patients with mCRPC within the study period. The mean age of the patients was 69 years. There was a biochemical confirmation of castration resistance in most of the patients (86.7\%). The mean serum Prostate Specific Antigen (PSA) at the time of diagnosis was $771 \mathrm{ng} / \mathrm{ml}$ and the mean Gleason Score was 8 . Antiandrogen withdrawal/substitution was the most common first line of management (72.4\%), while the use of docetaxel based chemotherapy $(36.8 \%)$ was the most common second line treatment. Only $13.3 \%$ were treated with the newer agents abiraterone and enzalutamide. Almost half of the patients (46.7\%) needed additional treatment with radiotherapy and/or zoledronic acid for symptomatic osseous metastases. Antiandrogen withdrawal/substitution was not significantly associated with increased risk of death at 18 months. Conclusion: Appropriate optimization and sequencing of the limited available treatment options for $\mathrm{mCRPC}$ are vital to a satisfactory outcome in a resource poor setting. Antiandrogen withdrawal/substitution should be a consideration in the management of mCRPC patients in resource poor environments.
\end{abstract}

Keywords: mCRPC Management, Antiandrogen Withdrawal, Chemotherapy, Resource Poor

\section{Introduction}

From being the eight most commonly diagnosed cancer in Nigerian men in the $1960 \mathrm{~s}$, carcinoma of the prostate (CAP) has now become the most common cancer in Nigerian men, accounting for $11 \%$ of all male cancers in Ibadan in the $1990 \mathrm{~s}$ as against $2.2 \%$ reported in the $1960 \mathrm{~s}$. [1, 2] The current combined age standardized incidence rate from two major national cancer registries in Nigeria is estimated as 19.1per 100,000 and hospital incidence rates of over 100per 100,000 have been reported. [3-5] Recently, a prevalence rate of 1046per 100, 000 was found amongst men that were screened for prostate cancer in communities in south western Nigeria. [6]

Late presentation of patients with carcinoma of the prostate is commonplace in Nigeria and most urologists in Nigeria are usually faced with managing mainly metastatic disease. [5] The initial treatment of choice for metastatic carcinoma of the prostate is usually some form of androgen deprivation therapy, commonly a bilateral orchidectomy in our low resource setting. While metastatic CAP often responds well to initial hormonal manipulation in the form of 
androgen deprivation therapy, most metastatic CAP invariably progress to a state of "hormone escape" - a phase in which the cancer is no longer responsive to the initial form of hormonal treatment/castration. [7] Formerly referred to as hormone refractory carcinoma of the prostate, hormone independent cancer or androgen independent cancer, this stage of the disease is now preferably and more appropriately termed castration resistant prostate cancer (CRPC). This is because the disease at this stage is still responsive to some other forms of hormonal manipulations. [8-11] Though the actual mechanism of development of castration resistance by the cancer cells is unknown, both androgen sensitive and androgen insensitive pathways have been described. [12]

While metastatic castration resistant prostate cancer (mCRPC) is traditionally regarded as a terminal disease with a median survival less than 2 years, there are currently new drugs that have been shown to improve survival in patients with mCRPC and these include docetaxel, abiraterone, enzalutamide, sipuleucel $\mathrm{T}$ and radium 223. [12] Some of these newer agents (though expensive) are becoming more available in Nigeria. The availability of multiple treatment options for mCRPC and lack of clear pragmatic local practice guidelines mean urologists and oncologists can be challenged with what should be the agent of choice and the sequencing of these agents, thus making clinical decision-making more complex. There is need for sharing experience to guide local practice towards achieving a cost effective method of the use of these agents in the treatment of $\mathrm{mCRPC}$ in resource poor regions.

We therefore describe our experience with the management of mCRPC at the Lagos State University Teaching Hospital Ikeja Lagos Nigeria.

\section{Patients and Methods}

The clinical records of all patients diagnosed with mCRPC at the Lagos State University Teaching Hospital, Ikeja Lagos over a 4 year period between June 2012 and June 2016 were retrospectively reviewed.

Data retrieved were patients' age, interval between diagnosis of metastatic carcinoma of the prostate and diagnosis of castration resistance, serum PSA and testosterone at diagnosis of mCRPC, the Gleason score, first line treatment given, second line treatment given, third line treatment given, additional treatment given, and mortality after eighteen months of follow up.

Data entry and analysis were done with Statistical Package for Social Sciences (SPSS) version 20.0 for Windows. The data expression was mainly with means and range. Tests for statistical significance were carried out using the Fischer's exact and Chi square test, with a $\mathrm{p}$ value $<0.05$ considered significant.

\section{Results}

There were 30 patients with $\mathrm{mCRPC}$ within the study period. The mean age of the patients was 69 years $(\mathrm{SD} \pm 9.2$ years). The range was $48-91$ years. The mean interval between the initial diagnosis of prostate cancer and diagnosis of castration resistance was 24.2 months (range 6-84 months). There was a biochemical confirmation of castration resistance in 26 patients $(86.7 \%)$. The diagnosis of castration resistance was clinical in 4 patients $(13.3 \%)$ who presented with worsening clinical condition with no available PSA at the initial time of diagnosis. The mean serum prostate specific antigen (PSA) at the time of diagnosis of castration resistance was $771 \mathrm{ng} / \mathrm{ml}$ (range $7.0-25,339 \mathrm{ng} / \mathrm{ml}$ ). The mean Gleason Score of the patients diagnosed with castration resistance was 8 (range $4-10$ ).

Antiandrogen withdrawal/substitution was the most common first line of management instituted in the patients (n $=21,70.0 \%$ ). The other first lines of management were use of docetaxel based chemotherapy in 7 patients $(23.3 \%)$, orchidectomy in 1 patient $(3.3 \%)$ and use of diethylstilbestrol in one patient $(3.3 \%)$. (Table 1 )

Table 1. First line treatment offered.

\begin{tabular}{ll}
\hline Treatment & Percentage (\%) \\
\hline Antiandrogen Substitution/Withdrawal & 70 \\
Docetaxel & 23.3 \\
Orchidectomy & 3.3 \\
Diethylstilbestrol & 3.3 \\
Total & 100 \\
\hline
\end{tabular}

Nineteen patients were already on second line treatment. Out of these, the use of docetaxel based chemotherapy ( $\mathrm{n}=7$, $36.8 \%$ ) was the most common second line treatment offered. Other second line treatment offered were use of ketoconazole in 6 patients $(31.6 \%)$, diethylstilbestrol in 4 patients $(21.1 \%)$ and the newer agents: abiraterone in one patient $(5.3 \%)$ and enzalutamide in one patient $(5.3 \%)$. (Table 2$)$

Table 2. Second line treatment offered.

\begin{tabular}{ll}
\hline Treatment & Percentage (\%) \\
\hline Docetaxel & 36.8 \\
Ketoconazole & 31.6 \\
Diethylstilbestrol & 21.1 \\
Abiraterone & 5.3 \\
Enzalutamide & 5.3 \\
Total & 100 \\
\hline
\end{tabular}

Out of the 5 patients who had commenced a third line treatment, 3 patients $(60 \%)$ were on ketoconazole and 2 patients $(40 \%)$ were on abiraterone. Overall only 4 patients $(13.3 \%)$ used the newer agents abiraterone and enzalutamide at any stage of treatment. Fourteen patients $(46.7 \%)$ had additional treatment with radiotherapy $+/$ - zoledronic acid for symptomatic osseous metastases.

There was a $10 \%(n=3)$ mortality rate as at eighteen months follow up period. There was no statistically significant difference in the mortality rates at eighteen months between the patients who had antiandrogen withdrawal/substitution as first line treatment and those who had chemotherapy or other agents $(p=0.636)$. There was however a statistically significant higher risk of death at eighteen months with the patients with PSA $>400 \mathrm{ng} / \mathrm{ml}$ 
compared with those with lower PSA values. $(p=0.048)$.

(Table 3)

Table 3. Association between nature of first line treatment, second line treatment and various PSA values with mortality at $18 m o n t h$.

\begin{tabular}{|c|c|c|c|c|}
\hline Parameters & & Alive & Dead & Pvalue \\
\hline \multirow{3}{*}{$1^{\text {st }}$ line treatment $n=29$} & Antiandrogen Withdrawal/Substitution & $19(90.5 \%)$ & $2(9.5 \%)$ & \multirow{3}{*}{$\mathrm{F}=1.047 P=0.636$} \\
\hline & Chemotherapy & $2(100 \%)$ & $0(0.0 \%)$ & \\
\hline & Others e.g. Ketoconazole & $5(83.3 \%)$ & $1(16.7 \%)$ & \\
\hline \multirow{4}{*}{$2^{\text {nd }}$ line treatment $n=21$} & Chemotherapy & $8(100 \%)$ & $0(0.0 \%)$ & \multirow{4}{*}{$\mathrm{F}=4.197 P=0.138$} \\
\hline & DES & $4(100 \%)$ & $0(0.0 \%)$ & \\
\hline & Ketoconazole & $6(85.7 \%)$ & $1(14.3 \%)$ & \\
\hline & Abiraterone/Enzalutamide & $1(50.0 \%)$ & $1(50.0 \%)$ & \\
\hline $\mathrm{PSA}>50$ at diagnosis & Not above 50 & $9(100 \%)$ & $0(0.0 \%)$ & $\chi^{2}=0.918 p=0.483$ \\
\hline \multirow{2}{*}{ PSA $>100$ at diagnosis } & Above 100 & $12(85.7 \%)$ & $2(14.3 \%)$ & \multirow{2}{*}{$\chi^{2}=2.449 p=0.209$} \\
\hline & Not above 100 & $16(100 \%)$ & $0(0.0 \%)$ & \\
\hline \multirow{2}{*}{ PSA $>400$ at diagnosis } & Above 400 & $5(71.4 \%)$ & $2(28.6 \%)$ & \multirow{2}{*}{$\chi^{2}=7.041 p=0.048$} \\
\hline & Not above 400 & $23(100 \%)$ & $0(0.0 \%)$ & \\
\hline
\end{tabular}

\section{Discussion}

Metastatic castration resistant prostate cancer is invariably terminal. The availability of multiple options of treatment means proper sequencing of the treatment is vital in optimizing the outcomes. This is particularly important as none of the available options is curative and the duration of response is limited. Though guidelines exists from the American Urological Association, European Association of Urology and other international bodies, there is often the need to modify these guidelines to reflect the realities of practice in resource poor areas where the vast majority of the patients pay out of pocket for very expensive cancer treatments. It is imperative that the various options of treatment are optimized in the best possible way to maximize patients' survival and quality of life on the one hand while making use of the limited funds available for patient care on the other. This is particularly important in our environment where the vast majority of our patients present with metastatic disease.

Most of our patients with mCRPC in this study presented with very high PSA values. Late presentation with prostate cancer in Nigeria had earlier been documented by other workers. $[5,13]$ The very high PSA recorded means that not only do most of our patients present late with metastases at the time of initial diagnosis of CAP, they also present late when they develop castration resistance. This is particularly unsettling, considering that these are patients who already have a diagnosis of cancer made and we presume that they will be compliant with their follow up appointments. The patients do not return to the hospital early enough when the castrate resistance can be diagnosed with a PSA rise alone but seem to only present when they have clinical evidence of advancing disease. The earliest manifestation of castration resistant prostate cancer is a steady rise in serum prostate specific antigen (PSA) after an initial drop. This rise in PSA usually will precede clinical evidence of advancing disease. [14] This stresses the potential benefits of patients' compliance with their follow up appointments for regular PSA checks, considering that we found a statistically significant increased risk of death amongst the patients with the higher PSA values.

Antiandrogen withdrawal was the most common first line treatment offered to our patients with mCRPC. Antiandrogen withdrawal or addition of another antiandrogen are recognized secondary hormonal treatments of mCRPC amongst others such as use of oestrogenic compounds and adrenolytic agents. [15, 16] Indeed some researchers recommend that antiandrogen withdrawal be done mandatorily before proceeding to other regimens in the treatment of mCRPC. [11] While these measures may not lead to increased overall survival, they do improve the progression free survival. In addition, antiandrogen withdrawal and/or addition help to delay the time of commencement of chemotherapy. [17] This advantage is important in our own resource poor environment where the newer options are quite expensive for fee paying patients. Though our study may have been underpowered, we did not find any statistically increased risk of death amongst the patients who had some form of antiandrogen withdrawal/substitution.

Docetaxel based chemotherapy was mostly used after the use of antiandrogen withdrawal or antiandrogen addition and this was the commonest second line treatment offered. Though the beneficial effect of chemotherapy is irrespective of age, pain or performance status at the time of initiation, [18], we generally tend to give chemotherapy only to our relatively younger mCRPC patients with very good performance status. This helps us to minimize potentially debilitating side effects in the very old or sick patients. With this approach, we have previously documented chemotherapy to be safe even in our busy local practice. [19]

Ketoconazole - a relatively cheap medication- is preferred as a second line hormonal treatment for our mCRPC patients who were unfit for chemotherapy. Ketoconazole has been shown to be beneficial in treatment of CRPC. [20] Ngo et al have recommended ketoconazole as a bridge in the treatment continuum of CRPC patients in whom chemotherapy may impact negatively on their quality of life. This is because of its low toxicity profile and affordability. [21]

Very few of our patients used the newer agents abiraterone 
and enzalutamide. This was due to the prohibitive costs of these agents in our environment and non-affordability by the patients. For the few patients who used these newer agents, they were used as second or third line options in our centre. These agents were well tolerated even by our very sick patients. While there are studies that now argue for the use/consideration of these newer agents as first line hormonal treatment for metastatic CAP - whether castrate resistant or hormone sensitive, $[22,23]$ this is yet to be the practice in our centre due to the non-affordability of these agents by most of the patients.

Vital to the management of mCRPC is the provision of palliative treatment measures especially for pain and spinal cord compression from symptomatic osseous metastases. The late presentation by our patients and the high volume of disease meant most of them had symptomatic osseous metastases with almost half of our patients needing external beam radiotherapy (EBRT) with steroids and regular zoledronic acid infusions. While surgical decompression can be carried out for spinal compression from mCRPC, ERBT and steroid can be offered as a satisfactory alternative treatment. [17] Zoledronic acid (4mg every 4 weeks) in $\mathrm{mCRPC}$ has been shown to significantly reduce the incidence of skeletal related events and to increase the time to the first skeletal related event and has a clinically significant effect in reduction of bone pain and total relief of pain. [24, 25] The need for additional treatment for most of our patients means a multidisciplinary approach to patient care for a successful outcome at this stage of the disease cannot be overemphasized.

Most of our patients survived for at least 18 months. Though earlier studies have suggested the adoption of a mainly palliative treatment for this stage of the disease in our environment [5], we argue that a more aggressive approach can be pursued with proper sequencing of the limited available treatment options with a decent outcome. Most of our patients are unable to afford the newer agents when they are recommended as options. The non-affordability of these newer agents (which are quite effective) for the treatment of mCRPC in resource poor areas calls for the need to modify some of the available international guidelines for use in local practice. There is the need for further research to guide urologists and oncologists on what should be the best and most cost effective treatment sequence for $\mathrm{mCRPC}$ in areas with limited resources.

\section{Conclusion}

Appropriate optimization and sequencing of the limited available treatment options for $\mathrm{mCRPC}$ are vital to a satisfactory/acceptable outcome. The use of antiandrogen withdrawal/substitution does not appear to impact negatively on the survival of mCRPC patients at least in the short term and thus should be a strong consideration in the treatment of mCRPC patients in resource poor environments with limited affordable treatment options.

\section{References}

[1] Nkposong EO, Lawani J. Primary carcinoma of the prostate in Ibadan. West African Medical Journal. 1973: 108-111.

[2] Ogunbiyi JO, Shittu OB. Increased incidence of prostate cancer in Nigerians. J Natl Med Assoc. 1999; 91 (3): 159-64.

[3] Jedy-Agba E, Curado MP, Ogunbiyi O et al. Cancer incidence in Nigeria: a report from population-based cancer registries. Cancer Epidemiol. 2012 Oct; 36 (5): e271-8. doi: 10.1016/j.canep.2012.04.007.Epub 2012 May 22.

[4] Osegbe DN. Prostate cancer in Nigerians: facts and nonfacts. J Urol. 1997; 157 (4): 1340-1343.

[5] Badmus TA, Adesunkanmi AR, Yusuf BM et al. Burden of prostate cancer in southwestern Nigeria. Urology 2010; 76 (2): 412-6.

[6] Ikuerowo SO, Omisanjo OA, Bioku MJ, Ajala MO, Mordi VP, Esho JO. Prevalence and characteristics of prostate cancer among participants of a community-based screening in Nigeria using serum prostate specific antigen and digital rectal examination. Pan Afr Med J. 2013 Aug 10; 15: 129. doi: 10.11604/pamj.2013.15.129.2489.eCollection 2013.

[7] Antonarakis ES, Carducci MA, Eisenberger MA. Treatment of Castration-Resistant Prostate Cancer. In: Wein AJ, Kavoussi LR, Novick AC, Partin AW, Peters CA, eds. Campbell-Walsh Urology. $10^{\text {th }}$ Edition. Philadelphia USA. 2012: 2954-2971.

[8] Murat F-J, Poissonnier L, Rabilloud M, et al. Mid-term results demonstrate salvage high-intensity focused ultrasound (HIFU) as an effective and acceptably morbid salvage treatment option for locally radiorecurrent prostate cancer. Eur Urol 2009; 55: 640-9.

[9] Scher HI, Halabi S, Tannock I, et al., Prostate Cancer Clinical Trials Working Group. Design and end points of clinical trials for patients with progressive prostate cancer and castrate levels of testosterone: recommendations of the Prostate Cancer Clinical Trials Working Group. J Clin Oncol 2008; 26: 1148-59.

[10] Scher HI, Beer TM, Higano CS, et al. Prostate Cancer Foundation/Department of Defense Prostate Cancer Clinical Trials Consortium. Antitumour activity of MDV3100 in castration-resistant prostate cancer: a phase 1-2 study. Lancet 2010; 375: 1437-46.

[11] Small EJ, Vogelzang NJ. Second-line hormonal therapy for advanced prostate cancer: a shifting paradigm. J Clin Oncol. 1997 Jan; 15 (1): 382-8.

[12] Cookson MS, Roth BJ, Dahm P et al. American Urological Association (AUA) Guidelines. American Urological Association Education and Research, Inc 2015: 1-23.

[13] Ekwere PD, Egbe SN. The changing pattern of prostate cancer in Nigerians: current status in the southeastern states. J Natl Med Assoc. 2002 Jul; 94 (7): 619-27.

[14] Eisenberger M, Crawford E, McLoed D et al. The prognostic significance of prostate specific antigen in stage D2 prostate cancer: interim evaluation of Intergroup 0105. Proc Am Soc Clin Oncol 1995; 14: abstract 612. 
[15] D'Amico AV, Chen MH, Renshaw AA, et al. Interval to testosterone recovery after hormonal therapy for prostate cancer and risk of death. Int J Radiat Oncol Biol Phys 2009; 75: $10-5$.

[16] Di Lorenzo G, Buonerba C, Autorino R, et al. Castrationresistant prostate cancer: current and emerging treatment strategies. Drugs 2010; 70: 983-1000.

[17] Mottet N, Bellmunt J, Bolla $\mathrm{M}$ et al. EAU Guidelines on Prostate Cancer. Part II: Treatment of Advanced, Relapsing, and Castration Resistant Prostate Cancer. European Urology 59 (2011) 572-583.

[18] Fitzpatrick JM, Anderson J, Sternberg CN, et al. Optimizing treatment for men with advanced prostate cancer: expert recommendations and the multidisciplinary approach. Crit Rev Oncol Hematol. 2008; 68 (suppl 1): S9-22.

[19] Omisanjo OA, Doherty AF, Omorinde MO, Egharevba PA, Bioku MJ, Ikuerowo SO. Docetaxel based chemotherapy in the management of metastatic castrate resistant prostate cancer in a Nigerian tertiary health institution. Nigerian Journal of Urology. 2015; 5 (1, 2): 14-16.

[20] Small EJ, Halabi S, Dawson NA et al. Antiandrogen withdrawal alone or in combination with ketoconazole in androgen-independent prostate cancer patients: a phase III trial (CALGB 9583). J Clin Oncol. 2004 Mar 15; 22 (6): 1025-33.

[21] Ngo LS, Yeo A, Wong AS, Tay MH. Efficacy of low-dose ketoconazole in hormone refractory prostate cancer patients at the National Cancer Centre and The Cancer Institute, Singapore. Ann Acad Med Singapore. 2007 Oct; 36 (10): 8114.

[22] Fizazi K, Tran N, Fein L et al. Abiraterone plus Prednisone in Metastatic, Castration-Sensitive Prostate Cancer. N Engl J Med. 2017 Jul 27; 377 (4): 352-360. doi: 10.1056/NEJMoa1704174. Epub 2017 Jun 4.

[23] Elkon JM, Millett RL, Millado KF, Lin J. Abiraterone is effective and should be considered for the treatment of metastatic castrate-naïve prostate cancer. Expert Opin Pharmacother. 2018 Apr; 19 (5): 507-509. doi: 10.1080/14656566.2018.1444028.Epub2018Mar1.

[24] Saad F, Gleason DM, Murray R et al. A randomized, placebo controlled trial of zoledronic acid in patients with hormone refractory metastatic prostate carcinoma. J Natl Cancer Inst 2002; 94: 1458-68.

[25] Kantoff PW, Higano CS, Shore ND et al. IMPACT Study Investigators. Sipuleucel-T immunotherapy for castrationresistant prostate cancer. N Engl J Med 2010; 363: 411-22. 